\title{
ON A GENERALIZATION OF A WAITING TIME PROBLEM AND SOME COMBINATORIAL IDENTITIES
}

\author{
B. S. EL-DESOUKY, ${ }^{* * *}$ \\ F. A. SHIHA * AND \\ A. M. MAGAR, ${ }^{*}$ Mansoura University
}

\begin{abstract}
In this paper we give an extension of the results of the generalized waiting time problem given by El-Desouky and Hussen (1990). An urn contains $m$ types of balls of unequal numbers, and balls are drawn with replacement until first duplication. In the case of finite memory of order $k$, let $n_{i}$ be the number of type $i, i=1,2, \ldots, m$. The probability of success $p_{i}=n_{i} / N, i=1,2, \ldots, m$, where $n_{i}$ is a positive integer and $N=\sum_{i=1}^{m} n_{i}$. Let $Y_{m, k}$ be the number of drawings required until first duplication. We obtain some new expressions of the probability function, in terms of Stirling numbers, symmetric polynomials, and generalized harmonic numbers. Moreover, some special cases are investigated. Finally, some important new combinatorial identities are obtained.
\end{abstract}

Keywords: Stirling number; generating function; waiting time; symmetric polynomial; harmonic number

2010 Mathematics Subject Classification: Primary 60C05; 05A10

Secondary 05A19; 11B73; $11 \mathrm{C} 08$

\section{Introduction}

An urn contains $m$ distinguishable balls which are sampled one at a time with replacement. The sampling is continued until the first duplication. Let $Y_{m}$ be the number of drawings required. This problem, which was solved by McCabe [10], is a special case of the problem of the waiting time until first duplication with finite memory of order $k$ of the preceding balls drawn. Let $Y_{m, k}$ be the number of draws required when there are $m$ different balls in the urn and there is finite memory of order $k$. The distribution of $Y_{m, k}$ was found by Arnold [1]. El-Desouky and Hussen [8] derived the following two cases.

Case 1. We generalize McCabe [10] as follows. Suppose that we have an urn containing $m$ types of balls with $n_{i}$ the number of balls of type $i, i=1,2, \ldots, m$. Assume that balls are sampled one at a time with replacement and the sampling is continued until the first duplication (i.e. until a ball of the same type has been drawn twice) and $Y_{m}$ is the number of drawings performed.

Remark 1. Case 1 can be considered as a special case of the problem of the waiting time until first duplication with finite memory of order $k$. In this case sampling is continued until a ball is drawn to duplicate one of the $k$ immediately preceding balls (one of each type) drawn. For example, when $k=1$, sampling stops only when two successive drawings yield a ball of the same type.

Received 3 June 2014; revision received 31 October 2014.

* Postal address: Department of Mathematics, Faculty of Science, Mansoura University, 35516 Mansoura, Egypt.

** Email address: b_desouky@yahoo.com 
Case 2. Let $\mathrm{Y}_{m, k}$ be the number of draws required when there are $m$ types of balls of unequal numbers and there is a finite memory of order $k$, which can be considered as a generalization of Arnold's problem [1]. It is clear that $Y_{m}$ is identical with $Y_{m, m}$ and if $k>m$, then $Y_{m, k}$ also has the same distribution as $Y_{m, m}$.

In this paper we give an extension of the results given in [8]. Hence we have the following theorem.

Theorem 1. Suppose that $p_{i}=n_{i} / N, N=\sum_{i=1}^{m} n_{i}$ is the probability that a ball of type $i$ is drawn. The probability $\mathbb{P}\left(Y_{m, m}>j\right)$ is given by

$$
\mathbb{P}\left(Y_{m, m}>j\right)=\sum_{i=0}^{j} \frac{j !}{i !}(-1)^{j-i} \sum_{k_{1}+k_{2}+\cdots+k_{i}=j} \frac{1}{k_{1} k_{2} \ldots k_{i}} \prod_{l=1}^{i} p_{k_{l}}(m)
$$

where $p_{k}(m)=\sum_{i=1}^{m}\left(p_{i}\right)^{k}$.

Proof. The exponential generating function of $\mathbb{P}\left(Y_{m, m}>j\right), j=1,2, \ldots, m$ is given by (see [8])

$$
\sum_{j=0}^{m} \mathbb{P}\left(Y_{m, m}>j\right) \frac{t^{j}}{j !}=\prod_{i=1}^{m}\left(1+p_{i} t\right)=\exp \left(\ln \prod_{i=1}^{m}\left(1+p_{i} t\right)\right)=\exp \left(\sum_{i=1}^{m} \ln \left(1+p_{i} t\right)\right),
$$

since $\ln \left(1+p_{i} t\right)=\sum_{k=1}^{\infty}(-1)^{k-1}\left(\left(p_{i} t\right)^{k} / k\right),\left|p_{i} t\right|<1$ implies that $|t|<1 / p_{i}$ and $0 \leq p_{i} \leq$ 1 , we have

$$
\sum_{j=0}^{m} \mathbb{P}\left(Y_{m, m}>j\right) \frac{t^{j}}{j !}=\exp \sum_{i=1}^{m}\left(\sum_{k=1}^{\infty}(-1)^{k-1} \frac{\left(p_{i} t\right)^{k}}{k}\right)=\exp \left(\sum_{k=1}^{\infty} \frac{(-1)^{k-1}}{k} t^{k} \sum_{i=1}^{m}\left(p_{i}\right)^{k}\right) .
$$

Let $\sum_{i=1}^{m}\left(p_{i}\right)^{k}=p_{k}(m)$; hence,

$$
\sum_{j=0}^{m} \mathbb{P}\left(Y_{m, m}>j\right) \frac{t^{j}}{j !}=\exp \left(\sum_{k=1}^{\infty} \frac{(-1)^{k-1}}{k} p_{k}(m) t^{k}\right)=\frac{\sum_{i=0}^{\infty}\left(\sum_{k=1}^{\infty}\left[(-1)^{k-1} / k\right] t^{k} p_{k}(m)\right)^{i}}{i !}
$$

Using the Cauchy rule of the product of a series, we obtain

$$
\begin{aligned}
\left(\sum_{k=1}^{\infty} \frac{(-1)^{k-1}}{k} p_{k}(m) t^{k}\right)^{i} & =\prod_{j=1}^{i}\left(\sum_{k_{j}=1}^{\infty} \frac{(-1)^{k_{j}-1}}{k_{j}} p_{k_{j}}(m) t^{k_{j}}\right) \\
& =\sum_{j=i}^{\infty} \sum_{k_{1}+k_{2}+\cdots+k_{i}=j} \frac{(-1)^{j-i}}{k_{1} k_{2} \ldots k_{i}} \prod_{l=1}^{i} p_{k_{l}}(m) t^{j}
\end{aligned}
$$

therefore,

$$
\begin{aligned}
\sum_{j=0}^{m} \mathbb{P}\left(Y_{m, m}>j\right) \frac{t^{j}}{j !} & =\sum_{i=0}^{\infty} \frac{1}{i !} \sum_{j=i}^{\infty} \sum_{k_{1}+k_{2}+\cdots+k_{i}=j} \frac{(-1)^{j-i}}{k_{1} k_{2} \ldots k_{i}}\left(\prod_{l=1}^{i} p_{k_{l}}(m)\right) t^{j} \\
& =\sum_{j=0}^{\infty} \sum_{i=0}^{j} \frac{1}{i !} \sum_{k_{1}+k_{2}+\cdots+k_{i}=j} \frac{(-1)^{j-i}}{k_{1} k_{2} \ldots k_{i}}\left(\prod_{l=1}^{i} p_{k_{l}}(m)\right) t^{j}
\end{aligned}
$$

Equating the coefficients of $t^{j}$ on both sides, we obtain (1). 
Remark 2. From [8, Equation (2.3)] and (1), we have the following new identity:

$$
s_{n}(m, m-j)=N^{j} \sum_{i=0}^{j} \frac{1}{i !}(-1)^{i} \sum_{k_{1}+k_{2}+\cdots+k_{i}=j} \frac{1}{k_{1} k_{2} \ldots k_{i}} \prod_{l=1}^{i} p_{k_{l}}(m),
$$

where $s_{n}(m, k)$ is the generalized Stirling number of the first kind associated with the real numbers $n_{1}, n_{2}, \ldots, n_{m}$ defined by (see [4], [5], and [7])

$$
\left(x-n_{1}\right)\left(x-n_{2}\right) \cdots\left(x-n_{m}\right)=\sum_{k=0}^{m} s_{n}(m, k) x^{k} .
$$

\section{Some special cases}

In what follows we discuss some special cases.

\subsection{Case 1}

Theorem 2. If $n_{i}=i, k=m$, the probability function $\mathbb{P}\left(Y_{m, m}>j\right)$ is given by

$$
\mathbb{P}\left(Y_{m, m}>j\right)=\sum_{i=0}^{j} \frac{j !}{i !} \sum_{k_{1}+k_{2}+\cdots+k_{i}=j} \frac{(-1)^{j-i}}{N^{j} k_{1} k_{2} \ldots k_{i}} \prod_{l=1}^{i} \sum_{r=1}^{k_{l}} r ! S\left(k_{r}, r\right)\left(\begin{array}{c}
m+1 \\
r+1
\end{array}\right),
$$

where $S(k, r)$ are the Stirling numbers of the second kind defined by (see [4])

$$
x^{n}=\sum_{i=0}^{n} S(n, i)(x)_{i}
$$

where $(x)_{i}=\prod_{l=0}^{i-1}(x-l)$.

Proof. If $n_{i}=i, p_{i}=i / N, i=1,2, \ldots, m$, and $N=\sum_{i=1}^{m} i=m(m+1) / 2$. From (3), we have

$$
\sum_{j=0}^{m} \mathbb{P}\left(Y_{m, m}>j\right) \frac{t^{j}}{j !}=\exp \left(\sum_{k=1}^{\infty} \frac{(-1)^{k-1}}{N^{k} k} t^{k} \sum_{i=1}^{m} i^{k}\right),
$$

since $\sum_{i=1}^{m} i^{k}=\sum_{r=1}^{k} S(k, r)\left(\begin{array}{c}m+1 \\ r+1\end{array}\right) r$ ! (see [2] and [12, p. 199]), we have

$$
\begin{aligned}
\sum_{j=0}^{m} \mathbb{P}\left(Y_{m, m}>j\right) \frac{t^{j}}{j !} & =\exp \left(\sum_{k=1}^{\infty} \sum_{r=1}^{k} r ! S(k, r)\left(\begin{array}{c}
m+1 \\
r+1
\end{array}\right) \frac{(-1)^{k-1}}{N^{k} k} t^{k}\right) \\
& =\sum_{i=0}^{\infty} \frac{1}{i !}\left(\sum_{k=1}^{\infty} \sum_{r=1}^{k} r ! S(k, r)\left(\begin{array}{c}
m+1 \\
r+1
\end{array}\right) \frac{(-1)^{k-1}}{N^{k} k} t^{k}\right)^{i}
\end{aligned}
$$

Using the Cauchy rule of the product of a series, we obtain

$$
\begin{aligned}
\left(\sum_{k=1}^{\infty} \sum_{r=1}^{k} r ! S(k, r)\right. & \left.\left(\begin{array}{c}
m+1 \\
r+1
\end{array}\right) \frac{(-1)^{k-1}}{N^{k} k} t^{k}\right)^{i} \\
& =\prod_{j=1}^{i}\left(\sum_{k_{j}=1}^{\infty} \sum_{r=1}^{k_{j}} r ! S\left(k_{j}, r\right)\left(\begin{array}{c}
m+1 \\
r+1
\end{array}\right) \frac{(-1)^{k_{r}+1}}{N^{k_{j}} k_{j}} t^{k_{j}}\right)
\end{aligned}
$$


therefore,

$$
\begin{aligned}
\sum_{j=0}^{m} \mathbb{P} & \left(Y_{m, m}>j\right) \frac{t^{j}}{j !} \\
& =\sum_{i=0}^{\infty} \frac{1}{i !} \sum_{j=i}^{\infty} \sum_{k_{1}+k_{2}+\cdots+k_{i}=j} \frac{(-1)^{j-i}}{N^{j} k_{1} k_{2} \ldots k_{i}}\left(\prod_{l=1}^{i} \sum_{r=1}^{k_{l}} r ! S\left(k_{l}, r\right)\left(\begin{array}{c}
m+1 \\
r+1
\end{array}\right)\right) t^{j} \\
& =\sum_{j=0}^{\infty} \sum_{i=0}^{j} \frac{1}{i !} \sum_{k_{1}+k_{2}+\cdots+k_{i}=j} \frac{(-1)^{j-i}}{N^{j} k_{1} k_{2} \ldots k_{i}}\left(\prod_{l=1}^{i} \sum_{r=1}^{k_{l}} r ! S\left(k_{l}, r\right)\left(\begin{array}{c}
m+1 \\
r+1
\end{array}\right) t^{j}\right.
\end{aligned}
$$

Equating the coefficients of $t^{j}$ on both sides, we obtain (4).

\subsection{Case 2}

Theorem 3. Let $n_{i}=n, k=m$, the probability function $\mathbb{P}\left(Y_{m, m}>j\right)$ is given by

$$
\mathbb{P}\left(Y_{m, m}>j\right)=\frac{(m)_{j}}{m^{j}}
$$

this is in agreement with [1].

Proof. Setting $n_{i}=n, i=1,2, \ldots, m$, i.e. there are an equal number of balls from each type, then $p_{i}=n / n m=1 / m$. From (3), we have

$$
\begin{aligned}
\sum_{j=0}^{m} \mathbb{P}\left(Y_{m, m}>j\right) \frac{t^{j}}{j !} & =\exp \left(\sum_{k=1}^{\infty} \frac{(-1)^{k-1}}{k} \sum_{i=1}^{m}\left(\frac{1}{m}\right)^{k} t^{k}\right) \\
& =\exp \left(m \sum_{k=1}^{\infty} \frac{(-1)^{k-1}}{k}\left(\frac{t}{m}\right)^{k}\right) \\
& =\exp \left(m \ln \left(1+\frac{t}{m}\right)\right) \\
& =\left(1+\frac{t}{m}\right)^{m} \\
& =\sum_{j=0}^{m}\left(\begin{array}{c}
m \\
j
\end{array}\right)\left(\frac{1}{m}\right)^{j} t^{j} .
\end{aligned}
$$

Equating the coefficients of $t^{j}$ on both sides yields (5).

\section{The asymptotic distribution of $Y_{m, m}$}

From (2) and using the first approximation $\ln \left(1+p_{i} t\right) \simeq p_{i} t$, where $p_{i} \rightarrow 0$ as $m \rightarrow \infty$, we have

$$
\sum_{j=0}^{m} \mathbb{P}\left(Y_{m, m}>j\right) \frac{t^{j}}{j !} \simeq \exp \left(\sum_{i=1}^{m} p_{i} t\right)=\exp (t)=\sum_{j=0}^{\infty} \frac{t^{j}}{j !}
$$

hence, for every $j \geq 1, \lim _{m \rightarrow \infty} \mathbb{P}\left(Y_{m, m}>j\right)=1$. 


\section{The distribution of $Y_{m, k}$ via a symmetric polynomial}

In this section we give a closed formula for the distribution of $Y_{m, k}$ by using the elementary symmetric polynomial for different cases. In the case of finite memory of order $k$, let $Y_{m, k}$ be the number of drawings required and $p_{i}=n_{i} / N, i=1,2, \ldots, m$.

Theorem 4. If $k=m, 1 \leq j \leq m$, then

$$
\begin{gathered}
\mathbb{E}\left(Y_{m, m}\right)=\sum_{j=2}^{m+1} j !\left(\sigma_{j-1}^{(m)}-j \sigma_{j}^{(m)}\right), \\
\operatorname{var}\left(Y_{m, m}\right)=\sum_{j=2}^{m+1} j j !\left\{\sigma_{j-1}^{(m)}-j \sigma_{j}^{(m)}\right\}-\left(\sum_{j=2}^{m+1} j !\left(\sigma_{j-1}^{(m)}-j \sigma_{j}^{(m)}\right)\right)^{2},
\end{gathered}
$$

where $\sigma_{j}^{(m)}\left(p_{1}, p_{2}, \ldots, p_{m}\right)$ is the elementary symmetric polynomial, defined in [9], by

$$
\sigma_{j}^{(m)}:=\sigma_{j}^{(m)}\left(p_{1}, p_{2}, \ldots, p_{m}\right)=\sum_{1 \leq i_{1}<i_{2}<\cdots<i_{j} \leq m} p_{i_{1}} p_{i_{2}} \ldots p_{i_{j}},
$$

and $\sigma_{j}^{(m)}=0$ for $j>m$ or $j<0$.

Proof. Since

$$
\begin{aligned}
\mathbb{P}\left(Y_{m, m}>j\right) & =\mathbb{P}(\text { the first } j \text { balls are all distinct, one of each type }) \\
& =j ! \sigma_{j}^{(m)}\left(p_{1}, p_{2}, \ldots, p_{m}\right),
\end{aligned}
$$

then the probability function of $Y_{m, m}$ is

$$
\begin{aligned}
\mathbb{P}\left(Y_{m, m}=j\right) & =\mathbb{P}\left(Y_{m, m}>j-1\right)-\mathbb{P}\left(Y_{m, m}>j\right) \\
& =(j-1) !\left(\sigma_{j-1}^{(m)}-j \sigma_{j}^{(m)}\right), \quad j=2,3, \ldots, m+1 .
\end{aligned}
$$

Hence, the mean of $Y_{m, m}$ is

$$
\begin{aligned}
\mathbb{E}\left(Y_{m, m}\right) & =\sum_{j=2}^{m+1} j \mathbb{P}\left(Y_{m, m}=j\right) \\
& =\sum_{j=2}^{m+1} j(j-1) !\left(\sigma_{j-1}^{(m)}-j \sigma_{j}^{(m)}\right) \\
& =\sum_{j=2}^{m+1} j !\left(\sigma_{j-1}^{(m)}-j \sigma_{j}^{(m)}\right) .
\end{aligned}
$$

This yields (6).

Equation (7) can be derived easily using (6) and the fact that

$$
\operatorname{var}\left(Y_{m, m}\right)=\mathbb{E}\left(\left(Y_{m, m}\right)^{2}\right)-\left(\mathbb{E}\left(Y_{m, m}\right)\right)^{2},
$$

and

$$
\mathbb{E}\left(\left(Y_{m, m}\right)^{2}\right)=\sum_{j=2}^{m+1} j^{2} \mathbb{P}\left(Y_{m, m}=j\right)=\sum_{j=2}^{m+1} j j !\left\{\sigma_{j-1}^{(m)}-j \sigma_{j}^{(m)}\right\}
$$


Note that there is another expression for the mean which is given by

$$
\mathbb{E}\left(Y_{m, m}\right)=\sum_{j=0}^{\infty} \mathbb{P}\left(Y_{m, m}>j\right)=\sum_{j=0}^{m} j ! \sigma_{j}^{(m)}
$$

(Note that $\sigma_{j}^{(m)}=0$ for $j>m$ ).

Theorem 5. If $k<m$, the probability $\mathbb{P}\left(Y_{m, k}>j\right)$ is given by

$$
\mathbb{P}\left(Y_{m, k}>j\right)= \begin{cases}j ! \sigma_{j}^{(m)}, & j=1,2, \ldots, k+1, \\ \left(\frac{(k+1) \sigma_{k+1}^{(m)}}{\sigma_{k}^{(m)}}\right)^{j-(k+1)}(k+1) ! \sigma_{k+1}^{(m)}, & j>k+1 .\end{cases}
$$

Proof. For $1 \leq j \leq k+1$,

$$
\mathbb{P}\left(Y_{m, k}>j\right)=j ! \sigma_{j}^{(m)}, \quad j=1,2, \ldots, k+1 .
$$

If $j>k+1$, then the conditional probability $\mathbb{P}\left(Y_{m, k}=j \mid Y_{m, k}>j-1\right)$ is equal to $\mathbb{P}[j$ th ball drawn has one of $k$ different types (those of the preceding $k$ balls)], so that

$$
\begin{aligned}
\mathbb{P}\left(Y_{m, k}=j \mid Y_{m, k}>j-1\right) & =\mathbb{P}\left(Y_{m, k}=k+1 \mid Y_{m, k}>k\right) \\
& =\frac{\mathbb{P}\left(Y_{m, k}=k+1\right)}{\mathbb{P}\left(Y_{m, k}>k\right)} \\
& =\frac{k ! \sigma_{k}^{(m)}-(k+1) ! \sigma_{k+1}^{(m)}}{k ! \sigma_{k}^{(m)}} .
\end{aligned}
$$

Thus,

$$
\begin{aligned}
\mathbb{P}\left(Y_{m, k}>j \mid Y_{m, k}>j-1\right) & =1-\mathbb{P}\left(Y_{m, k}=j \mid Y_{m, k}>j-1\right) \\
& =1-\frac{k ! \sigma_{k}^{(m)}-(k+1) ! \sigma_{k+1}^{(m)}}{k ! \sigma_{k}^{(m)}} \\
& =\frac{(k+1) ! \sigma_{k+1}^{(m)}}{k ! \sigma_{k}^{(m)}} .
\end{aligned}
$$

Hence,

$$
\begin{aligned}
\mathbb{P}\left(Y_{m, k}>j\right) & =\mathbb{P}\left(Y_{m, k}>j \mid Y_{m, k}>j-1\right) \mathbb{P}\left(Y_{m, k}>j-1\right) \\
& =\frac{(k+1) ! \sigma_{k+1}^{(m)}}{k ! \sigma_{k}^{(m)}} \mathbb{P}\left(Y_{m, k}>j-1\right) \\
& =\left(\frac{(k+1) ! \sigma_{k+1}^{(m)}}{k ! \sigma_{k}^{(m)}}\right)^{2} \mathbb{P}\left(Y_{m, k}>j-2\right) .
\end{aligned}
$$

By repeated application of this result, we obtain

$$
\mathbb{P}\left(Y_{m, k}>j\right)=\left(\frac{(k+1) ! \sigma_{k+1}^{(m)}}{k ! \sigma_{k}^{(m)}}\right)^{l} \mathbb{P}\left(Y_{m, k}>j-l\right) .
$$


Putting $j-l=k+1$, we have

$$
\mathbb{P}\left(Y_{m, k}>j\right)=\left(\frac{(k+1) \sigma_{k+1}^{(m)}}{\sigma_{k}^{(m)}}\right)^{j-(k+1)} \mathbb{P}\left(Y_{m, k}>k+1\right) .
$$

This yields (8).

From Theorem 5 we have the following corollary.

Corollary 1. The probability function of $Y_{m, k}$ is given by

$$
\begin{aligned}
\mathbb{P}\left(Y_{m, k}=\right. & j) \\
\quad= & \begin{array}{ll}
(j-1) !\left(\sigma_{j-1}^{(m)}-j \sigma_{j}^{(m)}\right), \\
(k+1) ! \sigma_{k+1}^{(m)}\left(\frac{(k+1) \sigma_{k+1}^{(m)}}{\sigma_{k}^{(m)}}\right)^{j-k-2}\left(\frac{\sigma_{k}^{(m)}-(k+1) \sigma_{k+1}^{(m)}}{\sigma_{k}^{(m)}}\right), & j>k+1 .
\end{array}
\end{aligned}
$$

\subsection{Special cases}

We derive another proof, using the elementary symmetric polynomials, of Arnold's results $[1$, Equation (5)] as follows.

Theorem 6. Let $n_{i}=n, k<m$, the probability $\mathbb{P}\left(Y_{m, k}>j\right)$ is given by (see [1])

$$
\mathbb{P}\left(Y_{m, k}>j\right)= \begin{cases}\frac{(m)_{j}}{m^{j}}, & j=1,2, \ldots, k+1, \\ \left(1-\frac{k}{m}\right)^{j-(k+1)} \frac{(m)_{k+1}}{m^{k+1}}, & j>k+1 .\end{cases}
$$

Proof. Setting $n_{i}=n, i=1,2, \ldots, m$, then $p_{i}=n / n m=1 / m$; hence,

$$
\sigma_{j}^{(m)}\left(p_{1}, p_{2}, \ldots, p_{m}\right)=\sigma_{j}^{(m)}\left(\frac{1}{m}, \frac{1}{m}, \ldots, \frac{1}{m}\right)=\left(\begin{array}{c}
m \\
j
\end{array}\right)\left(\frac{1}{m}\right)^{j},
$$

by substitution in (8), for $j=1,2, \ldots, k+1$, then

$$
P\left(Y_{m, k}>j\right)=j ! \sigma_{j}^{(m)}=j !\left(\begin{array}{c}
m \\
j
\end{array}\right)\left(\frac{1}{m}\right)^{j}=\frac{(m)_{j}}{m^{j}} .
$$

For $j>k+1$, we have

$$
\mathbb{P}\left(Y_{m, k}>j \mid Y_{m, k}>j-1\right)=\frac{(k+1) ! \sigma_{k+1}^{(m)}}{k ! \sigma_{k}^{(m)}}=\frac{(m)_{k+1}}{m^{k+1}} \frac{m^{k}}{(m)_{k}}=1-\frac{k}{m} .
$$

This yields (9).

Theorem 7. If $n_{i}=i, i=1,2, \ldots, m, k<m$, then

$$
\mathbb{P}\left(Y_{m, k}>j\right)= \begin{cases}\frac{j !}{N^{j}}(-1)^{j} s(m+1, m-j+1), & 1 \leq j \leq k+1, \\ \left(\frac{(k+1) s(m+1, m-k)}{s(m+1, m-k+1)}\right)^{j-(k+1)} & \\ \times \frac{(k+1) !}{N^{j}}(-1)^{j} s(m+1, m-k), & j>k+1,\end{cases}
$$


where $s(m, k)$ are the Stirling numbers of the first kind, defined by (see [5] and [11])

$$
(x)_{n}=\prod_{i=0}^{n-1}(x-i)=\sum_{i=0}^{n} s(n, i) x^{i} .
$$

Proof. Setting $n_{i}=i, i=1,2, \ldots, m$, then $p_{i}=i / N, N=m(m+1) / 2$; hence,

$$
\begin{aligned}
\sigma_{j}^{(m)}\left(p_{1}, p_{2}, \ldots, p_{m}\right) & =\sigma_{j}^{(m)}\left(\frac{1}{N}, \frac{2}{N}, \ldots, \frac{m}{N}\right) \\
& =\frac{1}{N^{j}} \sigma_{j}^{(m)}(1,2, \cdots, m) \\
& =\frac{1}{N^{j}}(-1)^{j} s(m+1, m-j+1),
\end{aligned}
$$

since $\sigma_{j}^{(m)}(1,2, \ldots, m)=(-1)^{j} s(m+1, m-j+1)$; see [5, Equation (5i), p. 214]. Substituting into (8), we obtain (10).

Note that for all the previous cases the mean $\mathbb{E}\left(Y_{m, k}\right)=\sum_{j=0}^{\infty} \mathbb{P}\left(Y_{m, k}>j\right)$ can be easily obtained.

\section{A new expression of $\mathbb{P}\left(Y_{m, m}>j\right)$}

Finally, we obtain a new expression of the probability $\mathbb{P}\left(Y_{m, m}>j\right)$ in terms of the generalized harmonic number.

Theorem 8. The probability $\mathbb{P}\left(Y_{m, m}>j\right)$ is given by

$$
\mathbb{P}\left(Y_{m, m}>j\right)=\sum_{r=0}^{j} \frac{j !}{r !}(-1)^{j-r} \sum_{k_{1}+k_{2}+\cdots+k_{r}=j} \frac{1}{k_{1} k_{2} \ldots k_{r}} \prod_{l=1}^{r} H_{m}\left(k_{l} ; \bar{\alpha}\right),
$$

where $H_{m}(k ; \bar{\alpha})$ is the generalized harmonic number defined by

$$
H_{n}(k ; \bar{\alpha})=\sum_{r=1}^{n} \frac{1}{\left(\alpha_{r}\right)^{k}}
$$

where $\bar{\alpha}=\left(\alpha_{1}, \ldots, \alpha_{n}\right)$; see [3] and [6].

Proof. From (3), letting $p_{i}=1 / \alpha_{i}$, we have

$$
\begin{aligned}
\sum_{j=0}^{m} \mathbb{P}\left(Y_{m, m}>j\right) \frac{t^{j}}{j !} & =\exp \left(\sum_{k=1}^{\infty} \frac{(-1)^{k-1}}{k} t^{k} \sum_{i=1}^{m}\left(\frac{1}{\alpha_{i}}\right)^{k}\right) \\
& =\exp \left(\sum_{k=1}^{\infty} \frac{(-1)^{k-1}}{k} H_{m}(k ; \bar{\alpha}) t^{k}\right) \\
& =\frac{\sum_{r=0}^{\infty}\left(\sum_{k=1}^{\infty}\left[(-1)^{k-1} / k\right] H_{m}(k ; \bar{\alpha}) t^{k}\right)^{r}}{r !}
\end{aligned}
$$


Using the Cauchy rule of the product of a series, this leads to

$$
\begin{aligned}
\sum_{j=0}^{m} \mathbb{P}\left(Y_{m, m}>j\right) \frac{t^{j}}{j !} & =\sum_{r=0}^{\infty} \frac{1}{r !} \sum_{j=r}^{\infty} \sum_{k_{1}+k_{2}+\cdots+k_{r}=j} \frac{(-1)^{j-r}}{k_{1} k_{2} \ldots k_{r}}\left(\prod_{l=1}^{r} H_{m}\left(k_{l} ; \bar{\alpha}\right)\right) t^{j} \\
& =\sum_{j=0}^{\infty} \sum_{r=0}^{j} \frac{1}{r !} \sum_{k_{1}+k_{2}+\cdots+k_{r}=j} \frac{(-1)^{j-r}}{k_{1} k_{2} \ldots k_{r}}\left(\prod_{l=1}^{r} H_{m}\left(k_{l} ; \bar{\alpha}\right)\right) t^{j} .
\end{aligned}
$$

Equating the coefficients of $t^{j}$ on both sides, we obtain (11).

Remark 3. From [8, Equation (2.3)] and (11), we have the following new identity:

$$
s_{\bar{\alpha}}(m, m-j)=N^{j} \sum_{r=0}^{j} \frac{(-1)^{r}}{r !} \sum_{k_{1}+k_{2}+\cdots+k_{r}=j} \frac{1}{k_{1} k_{2} \ldots k_{r}} \prod_{l=1}^{r} H_{m}\left(k_{l} ; \bar{\alpha}\right) .
$$

This identity provides us with a connection between the generalized Stirling number and the generalized harmonic number.

\section{Acknowledgement}

The authors would like to thank the anonymous referee for his/her valuable comments and suggestions.

\section{References}

[1] Arnold, B. C. (1972). The waiting time until first duplication. J. Appl. Prob. 9, 841-846.

[2] Beardon, A. F. (1996). Sums of power of integers. Amer. Math. Monthly 103, 201-213.

[3] Cakıć, N. P. (1995). The complete Bell polynomials and numbers of Mitrinović. Univ. Beograd. Publ. Elektrotehn. Fak. Ser. Math. 6, 74-78.

[4] Comtet, L. (1972). Nombres de Stirling généraux et fonctions symétriques. C. R. Acad. Sci. Paris A 275, A747-A750.

[5] Comtet, M. (1974). Advanced Combinatorics. Reidel, Dordrecht.

[6] EL-DesouKy, B. S. (2015). A generalization of Stirling and Lah numbers with some computational applications. To appear in ARS Combinatoria.

[7] El-Desouky, B. S. and Cakić, N. P. (2011). Generalized higher order Stirling numbers. Math. Comput. Modelling 54, 2848-2857.

[8] El-Desouky, B. S. And Hussen, S. A. (1990). A generalization of a waiting time problem. Adv. Appl. Prob. 22, 758-760.

[9] El-MikKawy, M. AND EL-Desouky, B. (2003). On a connection between symmetric polynomials, generalized Stirling numbers and the Newton general divided difference interpolation polynomial. Appl. Math. Comput. 138, 375-385.

[10] McCABE, B. (1970). Problem E2263. Amer. Math. Monthly 77, 1008.

[11] Riordan, J. (1968). Combinatorial Identities. John Wiley, New York.

[12] Rosen, K. H. (ed.) (2000). Handbook of Discrete and Combinatorial Mathematics. CRC Press, Boca Raton, FL. 